\title{
Transarterial Chemoembolization in Unresectable Hepatocellular Carcinoma with Portal Vein Tumor Thrombosis: A Tertiary Care Center Experience
}

\author{
Yashwant Patidar $^{1} \quad$ Basavaraj $^{1}$ Amar Mukund ${ }^{1}$ Shiv K. Sarin ${ }^{2}$ \\ ${ }^{1}$ Department of Interventional Radiology, Institute of Liver and \\ Biliary Sciences, New Delhi, India \\ ${ }^{2}$ Department of Hepatology, Institute of Liver and Biliary Sciences, \\ New Delhi, India \\ Address for correspondence Yashwant Patidar, MD, Department \\ of Interventional Radiology, Institute of Liver and Biliary Sciences, \\ D-1 Vasant Kunj, New Delhi, 110070, India
}

Indian J Radiol Imaging 2021;31:270-276.

\begin{abstract}
Keywords

- hepatocellular carcinoma

- portal vein tumor thrombosis

- transarterial chemoembolization

Background Portal vein tumor thrombosis (PVTT) is a common complication of hepatocellular carcinoma (HCC) occurring in 30 to $40 \%$ of cases. The presence of PVTT in $\mathrm{HCC}$ is regarded as an advanced disease that confers poor prognosis and survival. Transarterial chemoembolization (TACE) has traditionally been considered to be contraindicated in cases of PVTT, due to the risk of hepatic infarction, and further deteriorate liver function. We evaluated safety, technical efficacy, and outcomes of TACE in HCC with PVTT.

Methods From search results of the hospital database, out of 652 patients who underwent TACE for HCC, 73 patients of HCC with PVTT were retrospectively evaluated. Post-TACE tumor response by computed tomography (CT)/magnetic resonance imaging (MRI) imaging as per modified response evaluation criteria in solid tumors (mRECIST) criteria, if any occurrence of acute hepatic failure was assessed. Prognostic factors influencing survival were also determined.

Results In our study population, the mean age of the patients was 58 years. The 12and 24-month survival rates were 59 and 14\%, respectively, with an overall median survival of 12.3 months. A total of $58.9 \%$ patients had branch portal vein tumor thrombus and $41.1 \%$ had tumor thrombus in the main portal vein. We did not encounter any mortality or acute liver failure following TACE in a 30-day period. Both univariate and multivariate analysis revealed Child-Pugh score $(p=0.01)$ and the extent of tumoral thrombus ( $p$ 0.004) as a significant prognostic factor. Patients with branch PVTT, no ascites, and Child-Pugh A had better survival than those having main portal vein tumor thrombus, ascites, and Child-Pugh B.

Conclusion Our study concluded that TACE can achieve good disease control and improved survival in HCC with portal vein invasion despite being considered as a relative contraindication. Technical expertise, selection of patients, such as superselective catheterization and preserved liver function, are the key factors for a safe therapeutic procedure. Child-Pugh score and extent of portal vein invasion were the significant prognostic factors determining survival.
\end{abstract}

published online August 4, 2021
DOI https://doi.org/

$10.1055 / \mathrm{s}-0041-1734367$ ISSN 0971-3026

\author{
(C) 2021. Indian Radiological Association. \\ This is an open access article published by Thieme under the terms of the Creative \\ Commons Attribution-NonDerivative-NonCommercial-License, permitting copying \\ and reproduction so long as the original work is given appropriate credit. Contents \\ may not be used for commercial purposes, or adapted, remixed, transformed or \\ built upon. (https://creativecommons.org/licenses/by-nc-nd/4.0/). \\ Thieme Medical and Scientific Publishers Private Ltd. A-12, Second \\ Floor, Sector -2, NOIDA -201301, India
}




\section{Introduction}

Hepatocellular carcinoma (HCC) is one of the most common causes of malignancy-related deaths worldwide. The incidence is higher in Asian countries compared with Europe and the United States. ${ }^{1-3}$ The incidence of portal vein tumor invasion, in general, has been reported up to 30 to $40 \%$ in patients with HCC. ${ }^{4,5}$ The presence of portal vein tumor thrombus in HCC patients indicates a poorer prognosis reducing their overall survival to approximately 2 to 4 months with the best supportive care. ${ }^{6,7}$ Transarterial chemoembolization (TACE) has been recommended as the first line of management in unresectable HCC. As per the Barcelona Clinic Liver Cancer (BCLC) staging system, portal vein tumor thrombosis (PVTT) in HCC is considered as a relative contraindication for transarterial chemoembolization due to the potential risk of hepatic infarction and worsening liver function following embolization. Officially, BCLC staging system does not endorse intra-arterial therapy (IAT) in this group of patients, but certain studies have reported evidence supporting this treatment. ${ }^{8,9}$ The dual advantage of TACE is that it allows to administer locally high concentration of chemotherapeutic drugs directly to the tumor and thus reducing the systemic toxicity of these agents. Recent advances in technology and the use of micro catheters for superselective catheterization of arteries have given an edge to perform TACE in patients of HCC with PVTT. ${ }^{9}$ Few studies including meta-analyses have reported that TACE can be performed safely for HCC with PVTT with survival benefits in selected Child-Pugh class-A and -B patients. ${ }^{10,11}$ The current study was undertaken with the objectives of evaluating the efficacy, safety, and survival outcomes of TACE in HCC patients complicated by portal vein tumor thrombus.

\section{Study Population and Methods}

\section{Patient Selection}

This is a retrospective study done at a dedicated hepatobiliary tertiary care teaching hospital. The study was approved by the institutional review board. We searched through the hospital database system for patients with HCC who underwent TACE between 2011 and 2019. There were a total of 653 patients with unresectable HCC treated with TACE, comprising of 1,032 TACE sessions. Out of which, 73 patients of HCC with PVTT were enrolled in this study (22 patients received conventional TACE and 51 patients received drug-eluting beads TACE) ( - Fig. 1).

Inclusion criteria were serum bilirubin $<3 \mathrm{mg} / \mathrm{dL}$, aspartate aminotransferase (AST), or alanine aminotransferase (ALT) $<5$ times upper limit of normal, the Eastern Cooperative Oncology Group (ECOG) performance status $0-2$, and Child-Pugh A/B. Patients were excluded if they had surgical resection/liver transplantation/RFA/locoregional therapy/sorafenib or systemic chemotherapy prior to TACE, refractory ascites, clinical encephalopathy, extrahepatic metastasis, and complete portal vein thrombus extending up to splenic-superior mesenteric vein (SMV) confluence and contraindications to TACE.
All patients included in the study were biopsy proven and/or had triphasic imaging confirmation of the tumor. We reviewed baseline imaging and relevant laboratory parameters such as serum alpha-fetoprotein (AFP), complete blood count, and liver function tests. Portal vein tumor thrombus was interpreted on computed tomography (CT)/magnetic resonance imaging (MRI) by observing filling defects in the portal vein due to intraluminal mass causing expansion of portal vein with thread and streaks type of enhancement. We followed PVTT classification system developed by the Liver Cancer Study Group of Japan for assessing the degree of extent of PVTT $^{12}$ (Vp0: no tumor thrombus in the portal vein; $\mathrm{Vp} 1$ : presence of tumor thrombus distal to but not in the second-order branches of $\mathrm{PV} ; \mathrm{Vp2}$ : the second-order branches of PV; Vp3: first-order branches of the PV; Vp4: main trunk of the PV).

\section{Procedure}

Standard angiographic technique was used to access the common femoral artery. Celiac and mesenteric arteriograms were taken to assess arterial anatomy and tumor vascularisation. Superselective cannulation of the segmental and subsegmental hepatic artery branches feeding the tumor was done using a microcatheter (Progreat 2.7 Fr coaxial microcatheter system, Somerset, New Jersey, United States: Terumo Medical Corporation) and embolized with doxorubicin and Lipiodol emulsion (Guerbet, Paris, France) in 1:1 ratio or drug-eluting beads (Hepaspheres; Merit Medical Systems Inc.) of size 30 to $60 \mu \mathrm{m}$ loaded with $50 \mathrm{mg}$ of doxorubicin, which were mixed with nonionic iodinated contrast material in a ratio of $1: 1$. Any extrahepatic blood supply to the tumor (the inferior phrenic, intercostal arteries, or internal mammary), the respective arteries were cannulated and embolized in the same way. Stasis or near stasis (sluggish) of blood flow was considered as the end-point of embolization. After the procedure, the femoral arterial sheath was removed. Hemostasis was achieved through manual compression or vascular closure device.

\section{Postprocedure Assessment and Follow-up}

All patients had their serum bilirubin, AST, and ALT measured on days 2 to 5 post-TACE to monitor parenchymal injury and risk of acute liver failure as per CTCAE (common terminology criteria for adverse events version 4.0, for toxicities), any adverse events related to the procedure within 1 month were also sorted. All the patients were followed-up with contrast-enhanced CT or MRI done at 1,3, and 6, months, and annually thereafter. The treatment response was evaluated by the modified response evaluation criteria in solid tumors (mRECIST) criteria. Repeat TACE sessions were performed if the patients have residual tumor on imaging at 1 month and thereafter unless the patient had developed contraindication for TACE or showed absence of radiological response or AST $>25 \%$ elevation or worsening of Child-Pugh score. Follow-up of patients was done until their death or cut-off date of the study. 


\section{Statistical Methods}

Survival analysis was done by using the Kaplan-Meier method. All analyses were done using SPSS software version 22. Univariate and multivariate survival analyses were performed on selected variables, including Child-Pugh score, ascites status, ECOG performance status, and PVTT (branch vs. MPV), and $p$-value $<0.05$ was interpreted as statistically significant (-Fig. 1).

\section{Results}

Our study group comprised of 69 males and 4 females with a median age of presentation of 58 years ( - Table 1 ). The most common etiology was hepatitis B virus (HBV)-related infection accounting for $32.9 \%$ of cases. A total of $60 \%$ of patients had multifocal tumors. There were 49 and 24 patients of Child-Pugh classes A and B, respectively. Forty-eight patients had ascites. For the purpose of number and uniform comparison, we combined $\mathrm{Vp} 1$ to $\mathrm{Vp} 3$ as a branch portal vein and compared with $\mathrm{Vp} 4$ as the main portal vein thrombus. Tumor thrombus involving portal vein branches (Vp1-3) were 43 patients $(58.9 \%)$, and that involving the main portal vein was 30 (41.1\%). The average number of TACE sessions was two (range: 1-5). We also collected available protein-induced by vitamin-K absence II (PIVKA II) data from patients, only 22 patients had PIVKA II assay with a mean

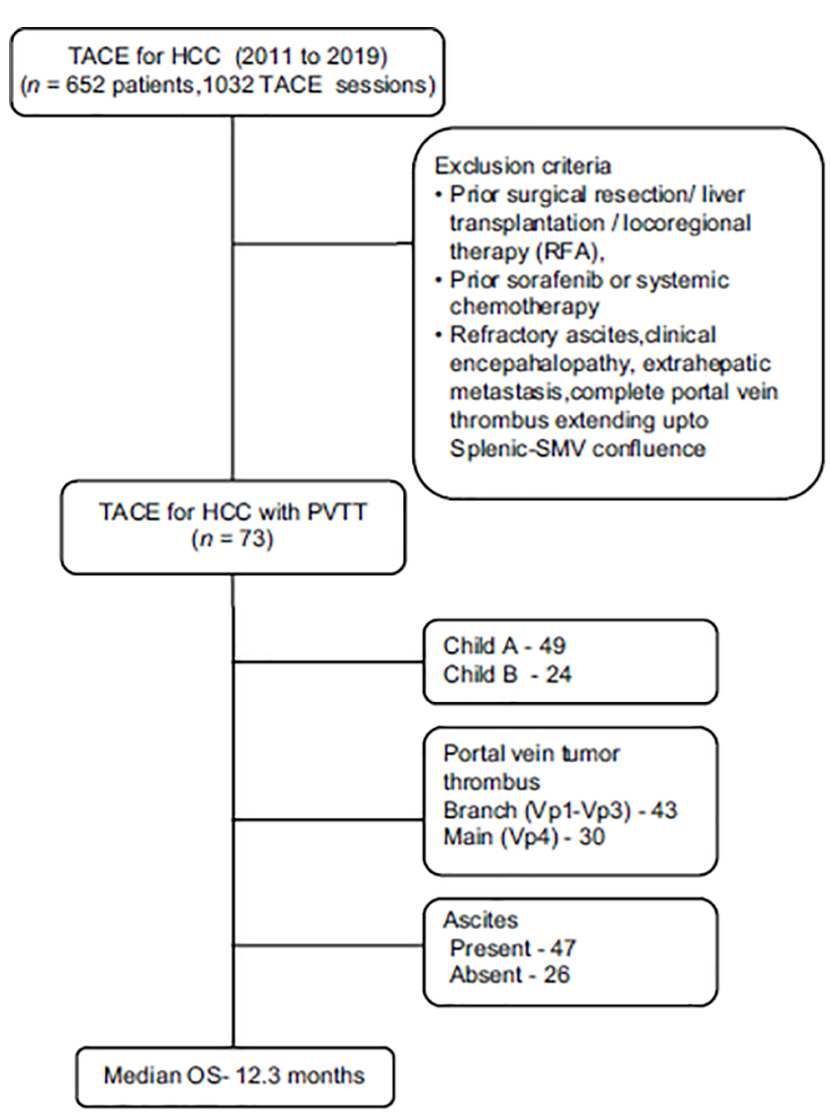

Fig. 1 Univariate and multivariate survival analyses were performed on selected variables as appropriate. HCC, hepatocellular carcinoma; OS, overall survival; PVTT, portal vein tumor thrombosis; SMV, superior mesenteric vein; TACE, transarterial chemoembolization; vp, Portal vein invasion; RFA, Radiofrequency ablatio. of $109.9 \mathrm{mAU} / \mathrm{mL}$. We found no procedure-related deaths or major complications such as acute liver failure/encephalopathy following TACE at 1 month. Minor adverse effects observed were related to postembolization syndrome such as fever (33\%), nausea and vomiting (21\%), abdominal pain (10\%), combined symptoms (17\%), transient elevation in liver enzymes (grades 2 and 3 as per CTCAE version 4.0; 14\%), and none (5\%). At 1-month follow-up, 3 patients showed complete response who had Vp1 type tumor thrombus (-Fig. 2), 67 of them showed partial response, and 3 patients showed stable disease (-Table 2). Meantime to tumor progression was 6.3 months $(6.3 \pm 2.8$ months $)$.

\section{Survival}

Overall survival rates at 12 and 24 months were 59 and 14\%, respectively, with a median survival of 12.3 months (95\% confidence interval $[\mathrm{CI}]$ : $11-14 ; p=0.01$; - Fig. 3). Survival analysis revealed significant correlation with child pugh score $(p=0.004)$ and branch versus MPV PVTT $(p=0.004)$ and ascites $(p=0.050)$. The median survival time of patients with Child-Pugh A versus B was $12.9 \pm 0.6$ and $9.2 \pm 1.01$ months $(p=0.004)$, respectively. The median survival time of patients with branch versus main PVTT was $13 \pm 1.1$ and $9.1 \pm$ 1.2 months ( $p=0.004)$, respectively. Patients without ascites had longer median survival time than patients with ascites (13 versus 10 months) with $p=0.050$ ( - Fig. 4).

\section{Prognosis Predictive Factors}

Univariate and multivariate survival analyses (- Table 3) were performed on selected variables including ascites status, Child-Pugh score and PVTT (branch vs. MPV), and ECOG performance status. Child-Pugh score $(p=0.004)$, (branch vs. MPV) PVTT ( $p=0.004)$, and ascites $(p=0.050)$ showed statistically significant correlation with survival in univariate analysis. However, on multivariate analysis Child-Pugh score

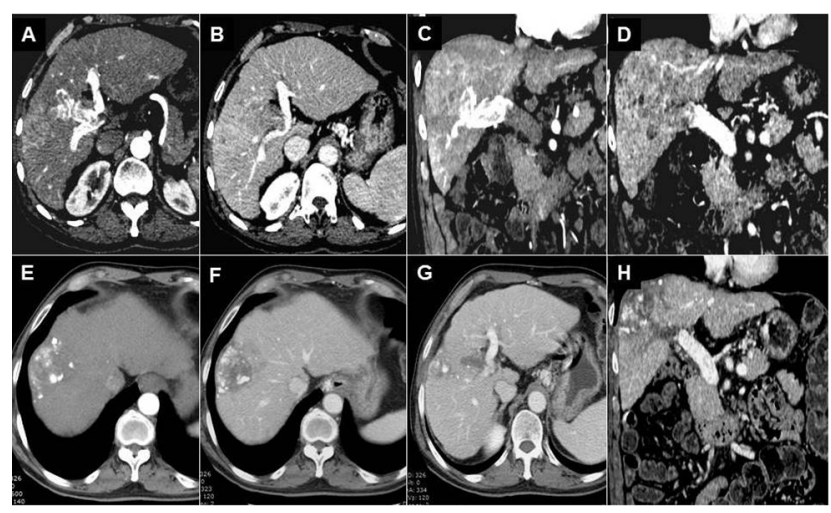

Fig. 2 (A-H) HCC with Vp1 type PVT: baseline CT images in the noncontrast axial phase image $(\mathbf{A})$, arterial phase axial $(\mathbf{B})$, portal venous phase axial (C), and portal venous phase coronal image (D) showing a large lesion in segment VIII of liver displaying arterial phase enhancement and venous phase washout suggestive of HCC with associate Vp1 PVT. Post-TACE follow-up CT noncontrast axial (E), arterial phase axial (F), portal venous phase axial (G), and portal venous phase coronal image $(\mathbf{H})$ showing complete lipiodol deposition and no any enhancement s/o complete response. CT, computed tomography; HCC, hepatocellular carcinoma; PVT, portal vein tumor; TACE, transarterial chemoembolization. 

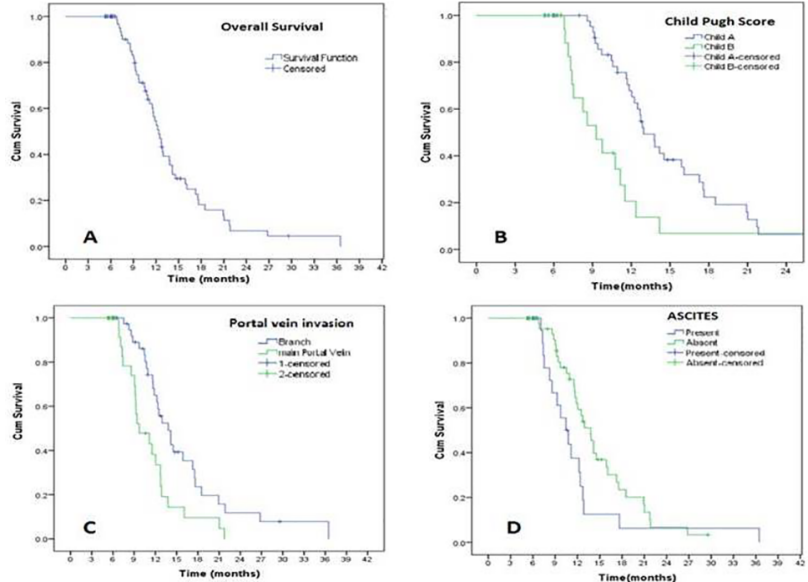

Fig. 3 (A-D): Kaplan-Meier survival curves: (A) overall survival, (B) branch versus main portal vein tumor thrombosis (PVTT), (C) ChildPugh $A$ versus $B$, and (D) ascites present versus absent.

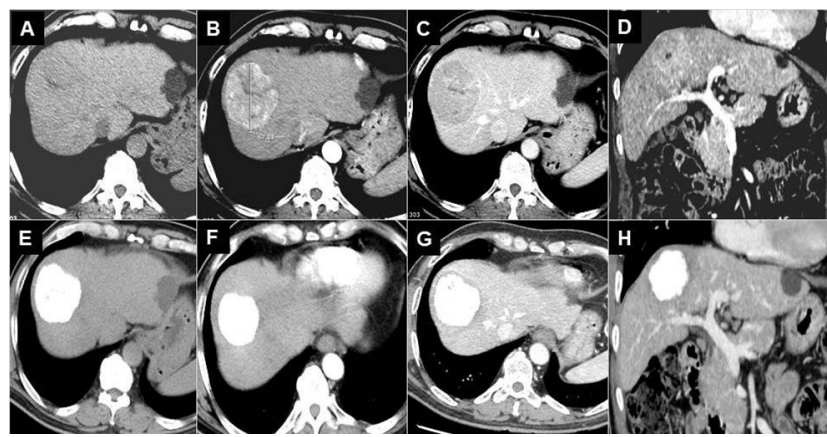

Fig. 4 (A-H) HCC with Vp3 type PVT: pre-TACE-Axial (A and B) and coronal (C and $\mathbf{D}$ ) images showing HCC in right lobe of liver invading portal vein $(\mathrm{VP} 3)$ with arterioportal shunting. Post-TACE follow-up 6 month CT Axial (E-G) and coronal $(\mathbf{H})$ images showing the reduced size of target lesion without any enhancement s/o complete response. CT, computed tomography; HCC, hepatocellular carcinoma; PVT, portal vein tumor; TACE, transarterial chemoembolization; vp,Portal vein invasion.

( $p=0.01)$ and (branch vs. MPV) portal vein tumor thrombus $(p=0.004)$ were significant prognostic predictive factors.

\section{Discussion}

HCC has a high propensity for vascular invasion. HCC with vascular invasion is classified as advanced stage. Portal vein invasion is the most common followed by hepatic veins and IVC invasion. ${ }^{13}$ HCC complicated by portal vein thrombosis presents as a challenging complication to treat as these patients have worsened liver function, compromised blood supply, and also they are at higher risk due to associated comorbid conditions such as portal hypertension. These patients have lesser tolerance to treatment and have a worse prognosis with a median survival time of 2 to 4 months with supportive management. ${ }^{14}$ The BCLC staging system considers PVTT as a relative contraindication for TACE and recommend sorafenib as a mainstay of therapy. This recommendation is based on the reason that liver is at increased
Table 1 Baseline characteristic of patients

\begin{tabular}{|c|c|}
\hline Variable & $\begin{array}{l}\text { Baseline } \\
\text { Mean } \pm \text { SD/n (\%) }\end{array}$ \\
\hline Age (y) & $58.5 \pm 11.2$ \\
\hline \multicolumn{2}{|l|}{ Sex } \\
\hline Male & $69(94.5)$ \\
\hline Female & $4(5.5)$ \\
\hline \multicolumn{2}{|l|}{ Etiology of cirrhosis } \\
\hline $\mathrm{HCV}$ & $11(15.1)$ \\
\hline HBV & $24(32.9)$ \\
\hline Ethanol & $16(21.9)$ \\
\hline $\mathrm{NASH}$ & $18(24.7)$ \\
\hline Cryptogenic & $4(5.5)$ \\
\hline \multicolumn{2}{|l|}{ ECOG PS } \\
\hline 0 & $37(50.7)$ \\
\hline 1 & $30(41.8)$ \\
\hline 2 & $6(8.2)$ \\
\hline \multicolumn{2}{|l|}{ Child class } \\
\hline$A$ & $49(67.1)$ \\
\hline $\mathrm{B}$ & $24(32.9)$ \\
\hline Average number of TACE sessions & 2 \\
\hline \multicolumn{2}{|l|}{ Tumor distribution } \\
\hline Solitary & $39(39.87)$ \\
\hline Multifocal & $44(60.3)$ \\
\hline \multicolumn{2}{|l|}{ Ascites } \\
\hline Yes & $48(67.1)$ \\
\hline No & $25(32.9)$ \\
\hline \multirow[t]{2}{*}{$\operatorname{AFP}(\mathrm{ng} / \mathrm{mL})$} & Pre-TACE: 1,210 (median) \\
\hline & Post-TACE: 850 (median) \\
\hline Serum bilirubin (mg/dL) & $1.26 \pm 0.5$ \\
\hline AST (IU/L) & $75.5 \pm 30.06$ \\
\hline $\operatorname{ALT}(\mathrm{IU} / \mathrm{L})$ & $50.4 \pm 23.9$ \\
\hline \multicolumn{2}{|l|}{ Portal vein invasion } \\
\hline Vp1 & $3(4.1)$ \\
\hline Vp2 & $15(20.5)$ \\
\hline Vp3 & $25(34.2)$ \\
\hline Vp4 & $30(41.9)$ \\
\hline
\end{tabular}

Abbreviations: AFP, alpha-fetoprotein; ALT, alanine aminotransferase; AST, aspartate aminotransferase; ECOG PS, Eastern Cooperative Oncology Group-performance status; HBV, hepatitis B virus; HCV, hepatitis C virus; SD, standard deviation; TACE, transarterial chemoembolization; NASH,Non-alcoholic steatohepatitis.

risk of liver infarction and liver failure following TACE in HCC with PVTT. ${ }^{15,16}$ Advances in technical skills and availability of superselective microcatheters have allowed to selectively target tumors and perform TACE sparing the uninvolved liver. Till date, very few studies have evaluated the safety and of TACE in HCC with PVTT. ${ }^{17-19}$ This study was undertaken to establish support, further strengthen and validate the evidence for the safety and efficacy of TACE in HCC with PVTT. Similar to Acharya, hepatitis B infection was the major cause 
of HCC in our study, reportedly being the most common cause of HCC in India. ${ }^{20}$ Comparison of our study outcomes was made with several studies in regard to the survival period ( - Table 4). Patients with Child-Pugh score A had better survival than Child-Pugh score B (13 vs. 9 months) similar to Georgiades et $\mathrm{al}^{21}$ whereas Chern et $\mathrm{al}^{22}$ reported better survival in patients without ascites compared with those with ascites. In our study also, we found improved survival in patients presenting without ascites than with ascites (13 vs. 10 months). A meta-analysis by Silva et $a l,{ }^{10}$ showed

Table 2 mRECIST response rates

\begin{tabular}{|l|l|}
\hline mRECIST response & Frequency \\
\hline CR & $3(4.1 \%)$ \\
\hline PR & $67(91.7 \%)$ \\
\hline SD & $3(4.1 \%)$ \\
\hline Total & 73 \\
\hline
\end{tabular}

Abbreviations: mRECIST, modified response evaluation criteria in solid tumors; SD, standard deviation; CR, Complete response; PR,Partial response. patients with tumor thrombus involving MPV had poor survival compared with branch portal vein. Similarly, in our study, patients with branch portal vein involvement had better survival than tumor thrombosis involving the main portal vein (13 vs. 9.1 months). Our analysis in these 73 patients revealed significant improvement of 1 -year survival rate (59\%) with a median survival of the entire group of 12.3 months similar to the study done by Kim et al ${ }^{23}$; Yoo et $\mathrm{al}^{24}$ reported favorable outcomes in patients without extrahepatic metastasis that partly explains improved median overall survival in our study, as we had excluded patients with extrahepatic metastasis. Postprocedure treatment response in all cases were done at 1 month with continued follow-up till death or study end period with cases having minimum of 6 months of follow-up. Our overall treatment response was based on mRECIST criteria given by Lencioni and Llovet. ${ }^{25}$ Three of our patients with Vp1 type of thrombus showed complete response; among them two showed progression at an interval of 8 and 16 months, while one showed no recurrence and progression-free survival for 2 years at

Table 3 Univariate and multivariate survival analysis

\begin{tabular}{|c|c|c|c|c|c|c|c|c|}
\hline & \multirow{2}{*}{$\begin{array}{l}\text { No of } \\
\text { patients }\end{array}$} & \multirow{2}{*}{$\begin{array}{l}\text { No of } \\
\text { deaths }\end{array}$} & \multicolumn{3}{|c|}{ Univariate analysis } & \multicolumn{3}{|c|}{ Multivariate analysis } \\
\hline & & & HR & $95 \% \mathrm{Cl}$ & $p$ & HR & $95 \% \mathrm{Cl}$ & $p$ \\
\hline \multicolumn{9}{|l|}{ Ascites } \\
\hline No & 47 & 34 & 1 & $0.9-3.3$ & 0.050 & 1 & $0.7-2.7$ & 0.7 \\
\hline Yes & 26 & 17 & 1.8 & & & 1.2 & & \\
\hline \multicolumn{9}{|l|}{ ECOG PS } \\
\hline 0 & 37 & 18 & 1 & $0.6-2.4$ & 0.46 & 1 & $0.6-2.3$ & 0.2 \\
\hline 1 & 30 & 27 & 1.2 & $0.8-6.1$ & 0.09 & 0.95 & $0.8-6.2$ & 0.1 \\
\hline 2 & 6 & 6 & 2.3 & & & 2.5 & & \\
\hline \multicolumn{9}{|l|}{ Child-Pugh } \\
\hline $\mathrm{A}$ & 48 & 35 & 1 & $1.3-4.5$ & $0.004^{\mathrm{a}}$ & 1 & $1.07-6.3$ & 0.01 \\
\hline B & 25 & 16 & 2.4 & & & 2.4 & & \\
\hline \multicolumn{9}{|l|}{ PVTT } \\
\hline Branch & 43 & 29 & 1 & $1.05-3.5$ & $0.004^{\mathrm{a}}$ & 1 & $1.3-4.3$ & 0.004 \\
\hline Main & 30 & 22 & 2.1 & & & 2.3 & & \\
\hline
\end{tabular}

Abbreviations: Cl, confidence interval; ECOG PS, Eastern Cooperative Oncology Group-performance status; HR, hazard ratio; PVTT, portal vein tumor thrombosis.

aStatistically significant correlation in both univariate and multivariate analysis.

Table 4 Comparative studies

\begin{tabular}{|c|c|c|c|c|c|}
\hline \multirow[t]{2}{*}{ Studies } & \multirow{2}{*}{$\begin{array}{l}\text { No. of } \\
\text { patients }\end{array}$} & \multirow{2}{*}{$\begin{array}{l}\text { Median OS } \\
\text { (mo) }\end{array}$} & \multicolumn{2}{|c|}{ Survival rates (\%) } & \multirow[t]{2}{*}{ Prognostic factors } \\
\hline & & & 1 year & 2 years & \\
\hline Georgiades et $\mathrm{al}^{21}$ & 32 & 11 & 25 & 12.5 & Child-Pugh score \\
\hline Chung et $\mathrm{a}^{29}$ & 110 & 6 & 30 & 18 & Tumor extent \\
\hline Chern et $\mathrm{a}^{22}$ & 50 & 6.2 & 22 & 10 & Ascites and response to treatment \\
\hline Kim et $a^{23}$ & 49 & 15 & 59 & 28 & Treatment type \\
\hline Yadav et $a^{30}$ & 17 & 10 & 47 & - & Child Pugh score and ascites \\
\hline Our study & 73 & 12.3 & 59 & 13 & Child-Pugh score, extent of portal vein invasion \\
\hline
\end{tabular}

Abbreviation OS, overall survival. 
follow-up. Sixty-seven patients had partial response and three patients had stable disease with varying lengths of time to tumor progression with a mean time to tumor progression of 6.3 months. Technically, the procedure was successful in all cases. Georgiades et $\mathrm{al}^{21}$ and Chung et $\mathrm{al}^{26}$ have postulated that there is a gradual formation of periportal collateral circulation and/or portal vein recanalization in these patients, thereby reducing the risk of acute liver failure. We did not encounter any mortality or acute liver failure following TACE in a 30-day period. No immediate procedure-related complications, like access site hematoma, pseudoaneurysm, Arteriovenous fistula, or arterial dissection, were reported. Postembolization syndrome was noted in majority of cases with transient elevation of liver enzymes upto grade 2.3 as per CTACE version 4.0; however, returned to near-baseline values in subsequent days with conservative management. Although recommendations suggest sorafenib as a targeted therapy in advanced HCC with portal vein invasion, the medial survival time with sorafenib is short which is around 6 months. ${ }^{27}$ Cho et al ${ }^{28}$ compared TARE versus sorafenib for HCC with PVT and found no significant difference in median overall survival and time to progression between two groups. Medial overall survival was (13.8 vs. 10 months) and time to progression was 6 versus 6 months. Our patients had a median overall survival of 12.3 months with time to progression of 6.3 months. Hence, we believe that transarterial chemoembolization is noninferior to TARE and better than sorafenib treatments with regard to survival and time to progression. TACE should be considered as the first-line treatment in patients of HCC with PVTT having good hepatic functional reserve without ascites which is safe and effective. Our study had few limitations like retrospective analysis and few patients received sorafenib treatment after TACE which could have influenced the obtained results.

\section{Conclusion}

To conclude, our study demonstrated that TACE can be safely performed in $\mathrm{HCC}$ with portal vein tumor thrombus and offers good disease control. The efficacy and survival outcomes of TACE in these patients are similar to other alternative treatment modalities described in the literature. Superselective catheterization technique and preserved liver function favor TACE as the first line of treatment in patients of HCC with PVTT. Child-Pugh score and extent of portal vein invasion are an important determinant of prognosis and survival.

\section{Financial Support and Sponsorship}

None.

\section{Conflicts of Interest}

There are no conflicts of interest.

\section{References}

1 Forner A, Llovet JM, Bruix J. Hepatocellular carcinoma. Lancet 2012;379(9822):1245-1255

2 Siegel R, Ma J, Zou Z, Jemal A. Cancer statistics, 2014. CA Cancer J Clin 2014;64(1):9-29
3 Global battle against cancer won't be won with treatment alone-effective prevention measures urgently needed to prevent cancer crisis. Cent Eur J Public Health 2014;22(1):23-28, 28

4 Yau T, Tang VY, Yao TJ, Fan ST, Lo CM, Poon RT. Development of Hong Kong Liver Cancer staging system with treatment stratification for patients with hepatocellular carcinoma. Gastroenterology 2014;146(7):1691-700.e3

5 Llovet JM, Ricci S, Mazzaferro V, et al; SHARP Investigators Study Group. Sorafenib in advanced hepatocellular carcinoma. N Engl J Med 2008;359(4):378-390

6 Llovet JM, Bustamante J, Castells A, et al. Natural history of untreated nonsurgical hepatocellular carcinoma: rationale for the design and evaluation of therapeutic trials. Hepatology 1999;29(1):62-67

7 Schöniger-Hekele M, Müller C, Kutilek M, Oesterreicher C, Ferenci P, Gangl A. Hepatocellular carcinoma in Central Europe: prognostic features and survival. Gut 2001;48(1):103-109

8 Kalva SP, Pectasides M, Liu R, et al. Safety and effectiveness of chemoembolization with drug-eluting beads for advanced-stage hepatocellular carcinoma. Cardiovasc Intervent Radiol 2014;37(2):381-387

9 Prajapati HJ, Dhanasekaran R, El-Rayes BF, et al. Safety and efficacy of doxorubicin drug-eluting bead transarterial chemoembolization in patients with advanced hepatocellular carcinoma. J Vasc Interv Radiol 2013;24(3):307-315

10 Silva JP, Berger NG, Tsai S, et al. Transarterial chemoembolization in hepatocellular carcinoma with portal vein tumor thrombosis: a systematic review and meta-analysis. HPB (Oxford 2017;19(8):659-666

11 Xue TC, Xie XY, Zhang L, Yin X, Zhang BH, Ren ZG. Transarterial chemoembolization for hepatocellular carcinoma with portal vein tumor thrombus: a meta-analysis. BMC Gastroenterol 2013;13:60

12 Liver Cancer Study Group of Japan, The General Rules for the Clinical and Pathological Study of Primary Liver Cancer. 3rd ed. Tokyo, Japan: Kanehara \& Co., Ltd; 2003

13 Chan SL, Chong CC, Chan AW, Poon DM, Chok KS. Management of hepatocellular carcinoma with portal vein tumor thrombosis: review and update at 2016. World J Gastroenterol 2016;22(32):7289-7300

14 Forner A, Reig ME, de Lope CR, Bruix J. Current strategy for staging and treatment: the BCLC update and future prospects. Semin Liver Dis 2010;30(1):61-74

15 Ye JZ, Zhang YQ Ye $\mathrm{HH}$, et al. Appropriate treatment strategies improve survival of hepatocellular carcinoma patients with portal vein tumor thrombus. World J Gastroenterol 2017; 17:141-147

16 Chung JW, Park JH, Han JK, et al. Hepatic tumors: predisposing factors for complications of transcatheter oily chemoembolization. Radiology 1996;198(1):33-40

17 Luo J, Guo RP, Lai EC, et al. Transarterial chemoembolization for unresectable hepatocellular carcinoma with portal vein tumor thrombosis: a prospective comparative study. Ann Surg Oncol 2011;18(2):413-420

18 Niu ZJ, Ma YL, Kang P, et al. Transarterial chemoembolization compared with conservative treatment for advanced hepatocellular carcinoma with portal vein tumor thrombus: using a new classification. Med Oncol 2012;29(4):2992-2997

19 Tawada A, Chiba T, Ooka Y, et al. Efficacy of transarterial chemoembolization targeting portal vein tumor thrombus in patients with hepatocellular carcinoma. Anticancer Res 2014;34(8):4231-4237

20 Acharya SK. Epidemiology of hepatocellular carcinoma in India. J Clin Exp Hepatol 2014;4(suppl 3) :S27-S33

21 Georgiades CS, Hong K, D’Angelo M, Geschwind JF. Safety and efficacy of transarterial chemoembolization in patients with unresectable hepatocellular carcinoma and portal vein thrombosis. J Vasc Interv Radiol 2005;16(12):1653-1659 
22 Chern MC, Chuang VP, Liang CT, Lin ZH, Kuo TM. Transcatheter arterial chemoembolization for advanced hepatocellular carcinoma with portal vein invasion: safety, efficacy, and prognostic factors. J Vasc Interv Radiol 2014;25(1):32-40

$23 \mathrm{Kim}$ JH, Yoon HK, Kim SY, et al. Transcatheter arterial chemoembolization vs. chemoinfusion for unresectable hepatocellular carcinoma in patients with major portal vein thrombosis. Aliment Pharmacol Ther 2009;29(12):1291-1298

24 Yoo JJ, Lee JH, Lee SH, et al. Comparison of the effects of transarterial chemoembolization for advanced hepatocellular carcinoma between patients with and without extrahepatic metastases. PLoS One 2014;9(11):e113926

25 Lencioni R, Llovet JM. Modified RECIST (mRECIST) assessment for hepatocellular carcinoma. Semin Liver Dis 2010;30(1):52-60

26 Chung GE, Lee JH, Kim HY, et al. Transarterial chemoembolization can be safely performed in patients with hepatocellular carcinoma invading the main portal vein and may improve the overall survival. Radiology 2011;258(2):627-634
27 Cheng AL, Kang YK, Chen Z, et al. Efficacy and safety of sorafenib in patients in the Asia-Pacific region with advanced hepatocellular carcinoma: a phase III randomised, double-blind, placebo-controlled trial. Lancet Oncol 2009;10(1):25-34

28 Cho YY, Lee M, Kim H-C, et al. Radioembolization Is a safe and effective treatment for hepatocellular carcinoma with portal vein thrombosis: A propensity score analysis. PLoS One 2016;11(5):e0154986

29 Chung JW, Park JH, Han JK, Choi BI, Han MC. Hepatocellular carcinoma and portal vein invasion: results of treatment with transcatheter oily chemoembolization. AJR Am J Roentgenol 1995;165(2):315-321

30 Yadav A, Hariprasad S, Gupta S, et al. Transarterial chemoembolization in unresectable hepatocellular carcinoma with portal vein thrombosis: a perspective on survival. Oman Med J 2014;29(6):430-436 